\title{
Mortality in people with schizophrenia in rural
}

\section{China}

\author{
10-year cohort study
}

\author{
MAO-SHENG RAN, ERIC YU-HAI CHEN, YEATES CONWELL, \\ CECILIA LAI-WAN CHAN, PAUL S. F. YIP, MENG-ZE XIANG \\ and ERIC D. CAINE
}

\begin{abstract}
Background Long-term mortality and the risk factors for premature death among patients with schizophrenia living in rural communities are unknown.
\end{abstract}

Aims To explore the 10 -year mortality and its risk factors among patients with schizophrenia.

Method We used data from a 10-year prospective follow-up study (1994-2004) of mortality among people with schizophrenia, and death registration data for Xinjin County, Chengdu, China.

Results The mortality rate was 2228 per 100000 person-years during followup. Both all-cause mortality and suicide rates were significantly greater in male than in female patients. Age at illness onset ( $>45$ years), duration of illness ( $\geqslant 10$ years), age greater than 50 years, physical illness, inability to work, male gender, and never having received treatment were identified as independent predictors of increased mortality.

Conclusions Higher mortality rates in male patients may contribute to the higher prevalence of schizophrenia in women compared with men in China. The findings of risk factors for mortality should be taken into account when developing interventions to improve outcomes among people with schizophrenia.

Declaration of interest None Funding detailed in Acknowledgements.
Although the mortality rate is higher among people with schizophrenia than among the general population (Mortensen \& Juel, 1993; Harris \& Barraclough, 1997; Harrison et al, 2001), little is known about the factors predicting death in these individuals (Salokangas et al, 2002). Most previous studies of mortality in schizophrenia were conducted in industrialised locales (Simpson \& Tsuang, 1996; Brown, 1997). Mortality in a broader range of cultures should be explored (Harrison et al, 2001; Simpson \& Tsuang, 1996). Mental health services are less available in rural China than in urban areas of the country (Xiang et al, 1994), possibly contributing to increased mortality rates there. However, studies of causes of death among people with schizophrenia in rural communities are sparse.

Although there are higher rates of schizophrenia in men than in women worldwide (Murray \& Lopez, 1996; Aleman et al, 2003), evidence indicates that there are substantially higher rates of schizophrenia in women than in men in China (Cooper \& Sartorius, 1996; Zhang et al, 1998; Liu et al, 2000; Ran et al, 2003; Phillips et al, 2004). The reasons why the pattern of schizophrenia in China differs from that in other parts of the world are unknown and largely unstudied.

Suicide is one of the most common causes of premature death in people with schizophrenia (Mortensen \& Juel, 1993; De Hert \& Peuskens, 2000). Given the unique pattern of suicide in China - rural rates are three times greater than urban rates, and rates in women are $25 \%$ higher than those for men in the general population (Phillips et al, 2002) - it is crucial to explore the characteristics of suicide in people with schizophrenia in rural China (Ran \& Chen, 2004). Previous studies have been limited by use of retrospective or cross-sectional designs, small numbers of patients, general problems of diagnostic standardisation, restricting the focus to in-patients only, and the relatively low rates of follow-up. Therefore, long-term prospective follow-up study of mortality in people with schizophrenia living in the community should be worthwhile (Harrison et al, 2001; Ran \& Chen, 2004).

Our study objectives were to explore the rates of all-cause mortality and suicide among people with schizophrenia, and to identify major causes of death and factors increasing the risk of death.

\section{METHOD}

\section{Study population}

The study sample consisted of people with schizophrenia $(n=510)$ identified in an epidemiological investigation of 123572 persons aged 15 years and older in six townships of Xinjin County in October 1994. Cases were identified through screening procedures for psychosis (face-to-face interviews with the head of each household, together with the key informant method) and general psychiatric interview. The details of this investigation have been described in previous papers (Ran et al, 2001, 2003). All participants lived in rural communities and met ICD-10 criteria (World Health Organization, 1992) for a diagnosis of schizophrenia based on standardised administration of the Present State Examination (PSE-9; Wing et al, 1974) by trained research interviewers. Using the baseline data acquired in 1994, we followed up and interviewed all the individuals with schizophrenia and their informants in May 2004. All respondents gave informed consent.

\section{Measurement}

The principal assessment tools included the PSE and Social Disability Screening Schedule (SDSS; Shen et al, 1986) used in the baseline investigation in 1994 (Ran et al, 2001, 2003). For cohort members who were alive when followed up in 2004 , at least one informant familiar with the person's life and circumstances and/or the cohort members themselves were interviewed. For those who had died, the next of kin or at least one informant familiar with the dead person was interviewed. All the interviews were conducted by trained psychiatrists using the Patients Follow-up Schedule (PFS) for about $30 \mathrm{~min}$; this questionnaire was used to collect information concerning demographic characteristics, cause and time of death, treatment and 
social support. For all cases, medical and psychiatric treatment records were obtained from hospital, village doctors' clinics and traditional healers. Information from the death certification and suicide note (where applicable) was also obtained. The classification of each death as due to suicide, accident or natural causes represented the consensus opinion of interviewers and independent researchers after reviewing all information obtained during the interviews. Participants were defined as homeless and lost to follow-up if an informant reported that they had wandered and slept in public places and that their whereabouts were now unknown. Participants' physical illnesses (e.g. heart disease, respiratory disease, cancer) at baseline and follow-up were defined according to the baseline data, informants' reports and doctors' diagnoses. Family economic status was defined according to the family mean income. Marked symptoms were defined according to the assessment of the PSE.

\section{Statistical analysis}

The follow-up period for every participant started at recruitment and ended at interview, death or the point when the inidividual was lost to follow-up. Mortality rates were calculated overall and by subgroups defined according to various characteristics. Mortality rates were estimated using the person-time method (number of deaths divided by person-years of follow-up). The effects of gender on all-cause mortality and suicide rates were tested using univariate Cox hazard regression analyses. Survival analyses were also used to explore gender differences in survival rates. Standardised mortality ratios were calculated by dividing observed deaths by expected deaths, with the general population of Xinjin County as the standard population. Death registration records for Xinjin County were used to derive data for the general population.

Predictors of mortality (crude mortality rates) were identified using univariate and multivariate Cox hazard regression analyses. Hazard ratios for potential predictors and the corresponding $95 \%$ confidence intervals were determined using univariate Cox regression. All variables with $P \leqslant 0.10$ in univariate analyses were included in a multivariate Cox model using a backwards procedure. Those with $P \leqslant 0.05$ were retained in the final model. The backwards procedure was used to exclude the potential

Table I Characteristics of the cohort in $1994(n=500)$

\begin{tabular}{lc}
\hline Characteristic & \\
\hline Gender, $n$ (\%) & $233(46.6)$ \\
$\quad$ Male & \\
Marital status, $n$ (\%) & $321(64.2)$ \\
$\quad$ Married & $104(20.8)$ \\
$\quad$ Single & $31(6.2)$ \\
$\quad$ Divorced & $279(55.8)$ \\
Family economic status below mean, $n$ (\%) & $141(28.2)$ \\
Family history of mental illness, $n$ (\%) & \\
Treatment history, $n$ (\%) & $105(21.0)$ \\
$\quad$ Previous hospitalisation & $240(48.0)$ \\
Medication & $153(30.6)$ \\
$\quad$ Never received treatment & $44.7(15.5)$ \\
Age, years: mean (s.d.) & $12.5(11.3)$ \\
Duration of illness, years: mean (s.d.) & $31.1(13.0)$ \\
Age at onset, years: mean (s.d.) &
\end{tabular}

confounding effect of variables by adding them, one at a time, in the final model. In addition, $2 \times 2$ interactions between independent predictors were tested.

In the Cox analyses and for estimation of the mortality and suicide rates, all independent variables other than gender were treated as time-dependent. All these variables were based on the measures at baseline or follow-up.

\section{RESULTS}

\section{Characteristics of the cohort}

Of the 510 people identified as having schizophrenia, 10 were excluded because they were lost to follow-up; therefore 500 people $(98.0 \%)$ were followed up from 1994 to 2004 . Their characteristics are described in Table 1. Half of this group were male, two-thirds were married and in $55.8 \%$ the family's economic status was less than the mean. One-fifth had been admitted to hospital and a third had never

Table 2 Status of the cohort patients in 2004

\begin{tabular}{lccc}
\hline & Men $(n=233)$ & Women $(n=267)$ & Total $(n=500)$ \\
& $n(\%)$ & $n(\%)$ & $n(\%)$ \\
\hline $\begin{array}{l}\text { Survivors } \\
\text { Deaths }\end{array}$ & $159(68.2)$ & $213(79.7)$ & $372(74.4)$ \\
$\quad$ Suicide & $15(6.4)$ & $6(2.3)$ & $21(4.2)$ \\
Death due to accident & $7(3.0)$ & $6(2.3)$ & $13(2.6)$ \\
$\quad$ Death due to natural causes & $36(15.5)$ & $28(10.5)$ & $64(12.8)$ \\
Homeless and lost to follow-up & $16(6.9)$ & $14(5.2)$ & $30(6.0)$ \\
\hline
\end{tabular}

received treatment at all. Of the 240 patients who accepted medication, 30 $(12.5 \%)$ were taking antipsychotic medication, $105(43.8 \%)$ maintained irregular antipsychotic medication for less than 2 months and 105 (43.7\%) had used only Chinese herbal medicine.

According to the investigation in 1994 (Ran et al, 2001, 2003) the incidence of schizophrenia was 0.58 per 1000 annually for men, and 0.57 per 1000 annually for women. The incidence risk ratio for men to develop schizophrenia relative to women was 1.01 (95\% CI $0.79-1.30, P>0.05)$. At the follow-up assessment in 2004, informants were available for all cases in the sample $(n=500)$. Information on 305 cases was provided by both participants themselves and informants, and information on 195 cases was provided by informants alone.

\section{Current status and mortality}

At the end of the follow-up period 372 $(74.4 \%)$ of the initial cohort were known 
to be alive (Table 2). The status of 30 people $(6.0 \%)$ who had been homeless was unknown to their family and friends. Twenty-one people $(4.2 \%)$ had died by suicide, $13(2.6 \%)$ had died due to accident and $64(12.8 \%)$ had died from natural causes during the follow-up period. Among all the 500 participants, $134(26.8 \%)$ had had various physical illnesses at baseline or at some time during the follow-up period, $11(2.2 \%)$ had migrated to cities for temporary work, and 6 women $(1.2 \%)$ had married and moved to other counties during the 10 years of follow-up.

The 98 deaths observed during followup represented a mortality rate of 2228 per 100000 person-years (95\% CI 17922664). Among men the mortality rate was 2913 (95\% CI 2174-3652) and among women it was 1661 (95\% CI 1150-2172). The rate was significantly higher in male than in female cohort members (hazard ratio $2.0,95 \%$ CI $1.3-3.2, P<0.005)$. The standardised mortality ratio for the whole cohort was 4.0 (95\% CI 2.4-5.8); for men it was 4.9 (95\% CI 2.8-8.1) and for women it was 3.3 (95\% CI 1.9-6.1).

Among those who died in the follow-up period, $21(21.4 \%)$ took their own lives, representing a suicide rate of 477 per 100000 person-years (95\% CI 273-681). Among men, the suicide rate was 753 per 100000 person-years (95\% CI 373-1133) and among women it was 249 per 100000 person-years (95\% CI 50-448). The rate was significantly higher in male than in female cohort members $(\mathrm{HR}=3.1$, $95 \%$ CI 1.2-8.0, $P<0.05)$. The standardised mortality ratio for all the cohort members who died by suicide was $32.0(95 \% \mathrm{CI}$ 18.5-52.5), for men it was 63.5 (95\% CI 43.6-94.5) and for women it was 13.4 (95\% CI 6.2-32.8). The 13 people who died as a result of accidents during the follow-up period represented a mortality rate due to accident of 296 per 100000 person-years (95\% CI 135-457). The standardised mortality ratio for the people who died by accidents was $6.6(95 \%$ CI 4.3-10.2). The 64 natural deaths during follow-up period represented a mortality rate due to natural causes of 1455 per 100000 person-years $(95 \%$ CI 11011809). The standardised mortality ratio for all the cohort members who died from natural causes was 2.6 (95\% CI 1.7-4.1). Among these 64 deaths, the specific causes of death in 42 cases $(66 \%)$ were known (various cancers, $n=9$; heart disease, $n=7$; respiratory disease, $n=7$; other disease, $n=19$ ).
The survival probability for the whole cohort in 2004 was 0.80 (95\% CI $0.76-$ 0.84). Compared with women, the survival rate of men during the 10-year follow-up was significantly lower (survival probability in 2004 for women, $0.84,95 \%$ CI $0.80-0.88$; survival probability in 2004 for men, $0.74,95 \%$ CI $0.68-0.80$; log-rank test $\chi^{2}=7.85, P<0.01$ ).

Mortality rates by clinical and demographic characteristics and results of the univariate and multivariate Cox regression analyses are presented in Table 3 . The independent predictors of mortality identified in the final model were age at onset of schizophrenia ( $>45$ years), duration of illness $(\geqslant 10$ years), age greater than 50 years, physical illness, inability to work, male gender, and never having received treatment. None of the variables excluded by the backwards procedure had a confounding effect.

\section{DISCUSSION}

To our knowledge this is the first 10 -year prospective cohort study on mortality among people with schizophrenia in rural China. It includes longitudinal follow-up and analyses based on time-dependent factors. The strengths of our study include the use of a large representative community sample in rural China, its longitudinal 10year follow-up design, and high rates of follow-up. Although studies of new-onset illness may accurately estimate suicide risk during the initial years of the illness (Palmer et al, 2005), study of a representative sample that includes both new-onset and chronic cases may better capture the death risk for the population of people with schizophrenia living in the community. As in most previous studies in China (Zhang et al, 1998; Phillips et al, 2004), all participants were 15 years or older at baseline in this study. It would not have substantially affected our results if children under 15 years old (a small group, representing less than $1 \%$ of all persons with schizophrenia) had been included.

\section{Mortality and suicide}

Compared with previous studies (Harris \& Barraclough, 1997; Osby et al, 2000; Harrison et al, 2001), our results showed that mortality and suicide rates are relatively high among people with schizophrenia, both men and women, in rural China. The overall mortality rate of 2228 per 100000 person-years observed in our study is extremely high, exceeding by 4 times the rate observed among people over 15 years old in the general population. This result is consistent with other results from low- and middle-income countries (Mojtabai et al, 2001). The suicide rate that we observed (477 per 100000 person-years) is close to the estimated rate in a previous study of people with schizophrenia in China $(0.68 \%$ per year; Phillips et al, 2004). Standardised mortality ratios were 32.0 for all suicide and 63.5 for males, which is much higher than in other countries (Osby et al, 2000). After excluding homeless individuals whose data were unavailable, we calculated the risk of suicide during the follow-up period as $4.5 \%$, which is close to the $4.9 \%$ risk found in a meta-analysis by Palmer et al (2005). Our study also indicated that the direct use of proportionate mortality rates in previous studies assuming a constant rate of suicide over a lifetime may overestimate suicide risk (Caldwell \& Gottesman, 1990). The rate of suicide in men with schizophrenia was significantly higher than that in women, which is consistent with a previous study (Phillips et al, 2004). Suicide rates among men and women with schizophrenia may be significantly influenced by the illness (e.g. onset, symptoms and treatment), which could be one explanation for the variation in suicide rates between people with schizophrenia and the general population.

\section{Mortality and prognosis}

Although some authors have suggested that there is a better prognosis for individuals with schizophrenia in low- and middleincome countries (Leff $e t a l, 1992$ ), our study showed a higher rate of mortality and missing due to homelessness among people with this disorder. It may be premature to suggest that there is a better prognosis for schizophrenia in these countries if withdrawals or attrition due to death and homelessness are not included in follow-up analyses. If the deceased and homeless cases were included in such analyses, the picture might change significantly. Deaths and homelessness among people with schizophrenia should be explored more in future natural history studies of this illness.

\section{Mortality and prevalence}

Different mortality rates in men and women in our study may explain the unique phenomenon of schizophrenia being more prevalent in women than in men in 
Table 3 Mortality rates and Cox regression analyses of mortality $(n=500)$

\begin{tabular}{|c|c|c|c|}
\hline Characteristic & $\begin{array}{c}\text { Deaths } \\
n\end{array}$ & $\begin{array}{l}\text { Mortality rate per } \\
100000 \text { person-years }\end{array}$ & $\begin{array}{l}\text { Hazard ratio } \\
(95 \% \mathrm{Cl})\end{array}$ \\
\hline \multicolumn{4}{|c|}{ Age at onset $>45$ years } \\
\hline Yes & 34 & 6415 & \multirow{2}{*}{$9.2(4.9-17.1)$} \\
\hline No & 64 & 1654 & \\
\hline \multicolumn{4}{|c|}{ Duration of illness $\geqslant 10$ years } \\
\hline Yes & 55 & 2517 & \multirow{2}{*}{$5.1(2.5-10.4)$} \\
\hline No & 43 & 1942 & \\
\hline \multicolumn{4}{|l|}{ Age $>50$ years } \\
\hline Yes & 64 & 2327 & \multirow[b]{2}{*}{$4.8(2.5-9.1)$} \\
\hline No & 34 & 2062 & \\
\hline \multicolumn{4}{|l|}{ Physical illness } \\
\hline Yes & 44 & 4015 & \multirow{2}{*}{$2.7(1.8-4.2)$} \\
\hline No & 54 & 1635 & \\
\hline \multicolumn{4}{|l|}{ Inability to work } \\
\hline Yes & 39 & 5571 & \multirow{2}{*}{$2.1(1.4-3.3)$} \\
\hline No & 59 & 1595 & \\
\hline \multicolumn{4}{|l|}{ Male gender } \\
\hline Yes & 58 & 2913 & \multirow[b]{2}{*}{$2.0(1.3-3.2)$} \\
\hline No & 40 & 1661 & \\
\hline \multicolumn{4}{|c|}{ Never received treatment } \\
\hline Yes & 40 & 3028 & \multirow[b]{2}{*}{$1.5(1.0-2.4)$} \\
\hline No & 58 & 1884 & \\
\hline \multicolumn{4}{|l|}{ Without income' } \\
\hline Yes & 55 & 4475 & \multirow{2}{*}{$1.5(0.9-2.6)$} \\
\hline No & 43 & 1357 & \\
\hline \multicolumn{4}{|c|}{ Family history of mental disorder ${ }^{2}$} \\
\hline Yes & 19 & $150 \mid$ & \multirow[b]{2}{*}{$1.5(0.9-2.5)$} \\
\hline No & 79 & 2522 & \\
\hline \multicolumn{4}{|l|}{ Married $^{2}$} \\
\hline Yes & 70 & 2169 & \multirow[b]{2}{*}{$1.3(0.9-2.1)$} \\
\hline No & 28 & 2389 & \\
\hline Living in shabby & & & \\
\hline Yes & 10 & 2674 & \\
\hline No & 88 & 2186 & $1.3(0.7-2.6)$ \\
\hline Number of fami & & & \\
\hline Yes & 48 & 2081 & \\
\hline No & 50 & 2390 & $1.2(0.8-1.9)$ \\
\hline Education $\leqslant 7 y$ & & & \\
\hline Yes & 81 & 2342 & \\
\hline No & 17 & 1809 & I.2(0.7-2.I) \\
\hline Family economi & & & \\
\hline Yes & 58 & 2344 & \\
\hline No & 40 & 2078 & $1.2(0.8-1.7)$ \\
\hline With marked sy & & & \\
\hline Yes & 67 & 2545 & \\
\hline No & 31 & 1755 & $1.2(0.8-1.8)$ \\
\hline Previous hospit & & & \\
\hline Yes & 20 & 1923 & \\
\hline No & 78 & 2322 & $1.2(0.7-1.9)$ \\
\hline Lives alone $^{2}$ & & & \\
\hline Yes & 23 & 2975 & \\
\hline No & 75 & 2068 & I.I (0.7-I.8) \\
\hline Previous suicide & & & \\
\hline Yes & 12 & 2013 & \\
\hline No & 86 & 2261 & $1.1(0.6-2.0)$ \\
\hline
\end{tabular}

I. Considered for the multivariate model $(P \leqslant 0.10$ in univariate analyses) but not retained.

2. Not considered for the multivariate model $(P>0.10$ in univariate analysis).
China. First, given that men have an earlier age at onset than women (Ran et al, 2003), the results of our study indicate that the higher mortality rate in men might be the major reason for the higher prevalence of schizophrenia in women than in men in China. Higher survival rates for female patients compared with male patients also support this opinion. This result may also explain in part why one finds more women among geriatric and late-onset patients with schizophrenia (Ran et al, 2004). Second, the results also indicated that women with schizophrenia were more likely to have married and moved away from the area. Even though the number is small, it is possible that female patients move to cities, which may increase the prevalence rates for women in urban areas. Third, our previous study did not support differences in the full remission rates between men and women (Ran et al, 2003).

All the risk factors identified in this study reflect the influence of both the socio-economic characteristics of rural China and the clinical characteristics of the cohort. Gender was an independent predictor of death during the follow-up period. Male gender was associated with an increased risk of death in this group, which is consistent with previous studies (Salokangas et al, 2002); the survival advantage of women held also among these participants. Why did the men have a significantly higher mortality rate than the women? Possible reasons may be that oestrogen has a protective effect in women (Seeman \& Lang, 1990), or that men with schizophrenia may accept less support and treatment than female patients in rural China (Ran et al, 2003). These possibilities warrant further study.

\section{Mortality and age}

Age ( $>50$ years) was an independent predictor of mortality during follow-up, as one would expect - older people with schizophrenia are much more likely to die (Palmer et al, 2005). Although young patients, early in the course of the illness, are more likely to attempt suicide than older patients, more patients may die from other causes with increasing age; the proportion who died by suicide is relatively small among all age groups. Our study showed that the mortality rate was significantly higher among people with later onset of schizophrenia ( $>45$ years) than among those with onset before 45 years of age. Although evidence indicates that individuals 
with a later onset may have a more benign illness course, symptom severity and cognitive deficits may be similar in both earlyonset and late-onset cases (Jeste et al, 1995). Although the finding that older patients and those with later-onset disorder have high mortality rates may not relate to lack of treatment, our study still suggests that higher mortality rates may be associated with the poor treatment received by older patients in rural China (Ran et al, 2004).

\section{Mortality and treatment}

Evidence indicates that a significant proportion of treated incident cases of schizophrenia achieve favourable long-term outcome (Harrison et al, 2001). Suicide risk among patients with schizophreniaspectrum disorders declines quickly after treatment and recovery (Qin \& Nordentoft, 2005). The results of our study indicated that never receiving treatment might increase the mortality rate among people with schizophrenia and, conversely, that treatment reduced mortality risk. In a $17-$ year follow-up study, the number of antipsychotic drugs taken by patients with schizophrenia showed a graded relation to mortality (Joukamaa et al, 2006). Given that certain classes of antipsychotic have been associated with death (Montout et al, 2002), we suggest that basic medication is important in decreasing the mortality and that excessive antipsychotic administration (e.g. overdose, multiple medication) may increase the mortality. The relationship between antipsychotics and mortality needs further study.

The results of our study indicate that long duration of illness and inability to work, which reflect poor social functioning, may increase the risk of mortality. The finding that previous hospitalisation is not a risk factor for mortality may be related to the lower rate of hospitalisation in rural China. Evidence indicates that people with schizophrenia have high rates of potentially reversible medical morbidity that additionally increase mortality (Green et al, 2003; Goff et al, 2005). In our study, over a quarter of the sample had physical illness, which predicted increased mortality. We suggest that treating medical comorbidity might reduce premature mortality among these patients (Goff et al, 2005).

\section{Implications for services}

Our results have implications for reducing mortality and suicide rates among people with schizophrenia in China and elsewhere. The risk factors of mortality should be taken into account when developing interventions to prevent premature death among these patients. Suicide prevention strategies should also be developed. Given the limited resources in contemporary China, prevention programmes should emphasise community-based mental healthcare to provide earlier diagnosis, antipsychotic treatment, treatment of comorbid medical conditions, function rehabilitation and family support. Given the severe stigma associated with psychiatric illness (Xiang et al, 1994), efforts to reduce stigma in the community will be necessary to enable individuals with schizophrenia to rejoin their community and allow interventions to be made to decrease their mortality rate.

Our findings indicate that homelessness among people with schizophrenia is a common phenomenon in rural China. Given that homeless individuals might experience mortality and suicide rates much higher than those of their counterparts in the general population (Roy et al, 2004), we suggest that suicide rates among people with schizophrenia might have been underestimated in previous studies in China (Phillips et al, 2004) because homeless people with the disorder were not included. Community-based mental health services, especially family and housing services, should be developed to prevent patients becoming homeless.

Given the representative sample used in this study, we are confident that our findings are generalisable to the population of people with schizophrenia in rural areas in China, and even to other countries that have a similar social environment. Premature death, suicide and homelessness are serious problems in people with schizophrenia in rural China. Supplying community mental health services and medication to these people should be a mental healthcare priority to prevent these early deaths.

\section{ACKNOWLEDGEMENTS}

The 1994 Chengdu study was supported by a grant from the China Medical Board in New York (CMB 92-557). This work was supported in part by the Hong Kong Jockey Club Centre for Suicide Research and Prevention, University of Hong Kong, and by International Clinical Operational and Health Services Research Training Award D43 TW058I4 (E.D.C.) and Global Research Initiative Program I ROI TW007260-0I (M.S.R.) from the Fogarty International Center, National Institutes of Health, USA.
The authors thank the Chengdu Mental Health Center (Wen-Jun Mao, MD, and Shi-Hui Hu, MD) and the Xinjin Mental Hospital (Cui-Ping Tang, RN, Fu-Rong Lin, MD, Li Li, MD and Li Si-Gan, MD) for their collaboration.

\section{REFERENCES}

Aleman, A., Kahn, R.S. \& Selton, J. (2003) Sex differences in the risk of schizophrenia. Archives of General Psychiatry, 60, 565-57I.

Brown, S. (1997) Excess mortality of schizophrenia: a meta-analysis. British Journal of Psychiatry, 17I, 502-508.

Caldwell, C. B. \& Gottesman, I. I. (1990)

Schizophrenics kill themselves too: a review of risk factors for suicide. Schizophrenia Bulletin, 16, 57|-588.

Cooper, J. E. \& Sartorius, N. (1996) Mental Disorders in China. Gaskell.

De Hert, M. \& Peuskens, J. (2000) Psychiatric aspects of suicidal behavior: schizophrenia. In International Handbook of Suicide and Attempted Suicide (eds K. Hawton, \& K. van Heeringen), pp. I2I-134. Wiley.

Goff, D. C., Cather, C., Evins, A. E., et al (2005) Medical morbidity and mortality in schizophrenia: Guideline for psychiatrists. Journal of Clinical Psychiatry, 66, 183-194.

Green, A. I., Canuso, C. M., Brenner, M. J., et al (2003) Detection and management of comorbidity in patients with schizophrenia. Psychiatric Clinics of North America, 26, I15-139.

Harris, E. C. \& Barraclough, B. (1997) Suicide as an outcome for mental disorders. A meta-analysis. British Journal of Psychiatry, 170, 205-228.

Harrison, G., Hopper, K., Craig, T., et al (2001) Recovery from psychotic illness: a 15- and 25-year international follow-up study. British Journal of Psychiatry 178, 506-517.

Jeste, D. V., Harris, M. J., Krull, A. J., et al (1995) Clinical and neuropsychological characteristics of patients with late-onset schizophrenia. American Journal of Psychiatry, 152, 722-730.

Joukamaa, M., Heliövaara, M., Knekt, P., et al (2006) Schizophrenia, neuroleptic medication and mortality. British Journal of Psychiatry, 188, 122-127.

Leff, J., Sartorius, N., Jablensky, A., et al (1992) The international pilot-study of schizophrenia: 5-year followup findings. Psychological Medicine, 22, I3I-145.

Liu, J. C., Wang, X. P. \& Yao, W. G. (2000)

Epidemiological survey of schizophrenia in Heilongjiang Province (in Chinese). Journal of Clinical Psychological Medicine, 10, 185

Mojtabai, R., Susser, E., Varma, V. K., et al (200I) Mortality and long-term course in schizophrenia with a poor 2-year course: a study in a developing country. British Journal of Psychiatry, 178, 7I-75.

Montout, C., Casadebaig, F., Lagnaoui, R., et al (2002) Neuroleptics and mortality in schizophrenia: prospective analysis of deaths in a French cohort of schizophrenic patients. Schizophrenia Research, 57, 147-156.

Mortensen, P. B. \& Juel, K. (1993) Mortality and causes of death in first admitted schizophrenic patients. British Journal of Psychiatry, 163, 183-189.

Murray, C. J. L. \& Lopez, A. D. (1996) Global Health Statistics: A Compendium of Incidence, Prevalence, and Mortality Estimates for Over 200 Conditions. Harvard University Press. 
Osby, U., Correia, N., Brandt, L., et al (2000) Mortality and causes of death in schizophrenia in Stockholm county, Sweden. Schizophrenia Research, 45, 2I-28.

Palmer, B. A., Pankratz, V. S. \& Bostwick, J. M. (2005) The lifetime risk of suicide in schizophrenia: A reexamination. Archives of General Psychiatry, 62, 247-253.

Phillips, M. R., Li, X.Y. \& Zhang, Y. P. (2002) Suicide rates in China, 1995-99. Lancet, 359, 835-840.

Phillips, M. R., Yang, G. H., Li, S. R., et al (2004) Suicide and the unique prevalence pattern of schizophrenia in mainland China: a retrospective observational study. Lancet, 364, 1062-1068.

Qin, P. \& Nordentoft, M. (2005) Suicide risk in relation to psychiatric hospitalization: evidence based on longitudinal registers. Archives of General Psychiatry, 62 427-432.

Ran, M. S. \& Chen, E. Y. H. (2004) Suicide and schizophrenia in China. Lancet, 364, 1016-1017.

Ran, M. S., Xiang, M. Z., Huang, M. S., et al (200I) Natural course of schizophrenia: two-year follow-up study in a rural Chinese community. British Journal of Psychiatry, 178, 154-158.

Ran, M. S., Xiang, M. Z., Li, S. X., et al (2003) Prevalence and outcome of schizophrenia in a Chinese rural area: an epidemiological study. Australian and New Zealand Journal of Psychiatry, 37, 452-457.

Ran, M. S., Xiang, M. Z., Conwell, Y., et al (2004) Comparison of characteristics between geriatric and younger subjects with schizophrenia in community. Journal of Psychiatric Research, 38, 417-424.

MAO-SHENG RAN, MD, PhD, Hong Kong Jockey Club Centre for Suicide Research and Prevention, University of Hong Kong, China, College of Professional Studies, University of Guam, USA, and Institute of Mental Health, West China Medical School of Sichuan University, Chengdu, China; ERIC YU-HAI CHEN, MD, Hong Kong Jockey Club Centre for Suicide Research and Prevention, Universisty of Hong Kong, China; YEATES CONWELL, MD, Department of Psychiatry, University of Rochester Medical Center, Rochester, New York, USA;

CECILIA LAI-WAN CHAN, PhD, PAUL S. F. YIP, PhD, Hong Kong Jockey Club Centre for Suicide Research and Prevention, University of Hong Kong; MENG-ZE XIANG, MD, Institute of Mental Health, West China Medical School of Sichuan University, Chengdu, China; ERIC D. CAINE, MD, Department of Psychiatry, University of Rochester Medical Center, Rochester, New York, USA

Correspondence: Dr Mao-Sheng Ran, College of Professional Studies, University of Guam, Mangilao, Guam 96923, USA. Tel: + I 67I 735 2655; fax: + I 67I 734 1203; email: ranmaosh@yahoo.com

(First received 8 April 2006, final revision 12 September 2006, accepted 27 October 2006)

Roy, E., Haley, N., Leclerc, P., et al (2004) Mortality in a cohort of street youth in Montreal. JAMA, 292 569-574.

Salokangas, R. K. R., Honkonen, T., Stengard, E., et al (2002) Mortality in chronic schizophrenia during decreasing number of psychiatric beds in Finland. Schizophrenia Research, 54, 265-275.

Seeman, M.V. \& Lang, M. (1990) The role of oestrogens in schizophrenia gender differences. Schizophrenia Bulletin, 16, 185-194.

Shen, Y. C., Chen, C. H., Zhang, W. X., et al (1986) An epidemiological investigation on mental disorders in 12 regions of China. Methodology and data analysis (in Chinese). Chinese Journal of Neurology and Psychiatry, 19, 65-69.
Simpson, J. C. \& Tsuang, M.T. (1996) Mortality among patients with schizophrenia. Schizophrenia Bulletin, 22 485-499.

Wing, J. K., Cooper, J. E. \& Sartorius, N. (1974) The Measurement and Classification of Psychiatric Symptoms. Cambridge University Press.

World Health Organization (1992) The ICD-10 Classification of Mental and Behavioural Disorders: Clinical Descriptions and Diagnostic Guidelines. WHO.

Xiang, M. Z., Ran, M. S. \& Li, S. G. (1994) A controlled evaluation of psychoeducational family intervention in a rural Chinese community. British Journal of Psychiatry, 165, 544-548.

Zhang, W. X., Shen, Y. C., Li, S. R., et al (1998) Epidemiological survey on mental disorders in 7 areas in China (in Chinese). Chinese Journal of Psychiatry, 31, 69-71. 